Short communication

\title{
Safety and pharmacokinetics of levobupivacaine with fascia iliaca compartment block in elderly patients
}

Peter M. Odor ${ }^{1}$, Alison G. Cavalier ${ }^{2}$, Neal Reynolds ${ }^{2}$, King S. Ang ${ }^{2}$, Simon J. Parrington ${ }^{2}$, Hua $\mathrm{Xu}^{3}$, Atholl Johnston ${ }^{4}$, Frederic Sage ${ }^{2}$

1. Department of Anaesthesia, St. George's University Hospital, London, UK

2. Department of Anaesthesia, East Surrey Hospital, Surrey, UK

3. Analytical Services International, St. George's University of London, UK

4. Department of Clinical Pharmacology, Barts and The London School of Medicine and Dentistry, Queen Mary University of London, London, UK

\author{
Address correspondence to: \\ Peter M. Odor, Department of Anaesthesia, St. George's University Hospital, London, UK. \\ email: peter.odor@nhs.net
}

ORCID 0000-0003-4642-2703

Running Head:

Levobupivacaine pharmacokinetics following fascia iliaca block 


\section{Key points}

- Use of fascia iliaca compartment block in elderly patients is safe, well tolerated and effective in reducing pain associated with a fractured neck of femur.

- Following administration of a $75 \mathrm{mg}$ dose of levobupivacaine via a fascia iliaca compartment block, systemic absorption of local anaesthetic into the plasma is slow ( $\mathrm{t}_{\max }$ 45 minutes).

- No relationship was observed between $\mathrm{C}_{\max }$ and $\alpha 1$ acid glycoprotein, weight, or body mass index. 


\section{Introduction}

Fascia iliaca compartment block (FICB) is a local anaesthetic block which provides unilateral analgesia to the lower limb [1]. FICB is an increasingly popular technique for the management of pain in elderly, frail patients with a fractured neck of femur, since the compartmental nerve block can provide effective opioid-sparing analgesia during the pre- and peri-operative period [2]. Although serious complications of FICB are rarely reported, this cohort of patients are particularly vulnerable to the risk of unrecognised local anaesthetic toxicity. This heightened risk is multifactorial: secondary to the increased prevalence of cognitive impairment in the elderly population, which may limit self-reporting of early toxicity symptoms; the pharmacodynamic effects of increasing age reducing clearance of amide local anaesthetics [3-5]; in addition to accelerated early absorption of local anaesthetic from the tissues [6]. Despite widespread use, there is currently very limited data on the pharmacokinetics of FICB and no data for FICB in elderly patients.

The primary aim of this study was to describe the pharmacokinetic profile of a standard, effective dose of levobupivacaine after FICB in elderly patients with hip fracture. As a secondary outcome measure, the relationship between levobupivacaine $C_{\max }$, plasma concentration of $\alpha 1$ acid glycoprotein (the primary binding protein of levobupivacaine) and patient anthropometric characteristics was also investigated. 


\section{Methods}

\subsection{Study design}

This single arm descriptive study was conducted in a single hospital in Surrey, England. The study was registered with the UK Clinical Trials Gateway (ISRCTN27364035) before onset of patient recruitment.

After obtaining written informed consent, we included 12 adults aged $\geq 80$ years, admitted with a diagnosis of fractured neck of femur who had consented to receive FICB as part of standard clinical care. Patients were excluded if they lacked capacity, had allergy to amide anaesthetics, renal failure (defined as serum creatinine $>300 \mu \mathrm{mol} / \mathrm{L}$ or receiving any form of renal replacement therapy) or weighed $<40 \mathrm{~kg}$ or $>120 \mathrm{~kg}$. All patients were recruited at a single district hospital in the UK.

All patients received a standardised dose and technique for FICB. The FICB procedure involves the aseptic injection of a large volume of local anaesthetic into the potential space between the iliacus muscle and covering layer of fascia iliaca. Ultrasound guided FICBs are performed away from major blood vessels and nerves to reduce the risk of intravascular injection or direct nerve injury. However, a relatively large volume of local anaesthetic is required to facilitate spread towards the nerves contained within the fascia iliaca compartment. Typically at least two of the three major nerves that supply the medial, anterior and lateral thigh are affected by local anaesthetic spread: most frequently the femoral nerve, lateral cutaneous nerve of the thigh and less frequently the obturator nerve.

Nerve blocks were all performed in the absence of sedation or general anaesthesia, in haemodynamically stable patients, none of whom were undergoing fluid resuscitation. Single shot blocks were provided by anaesthetic consultants or senior anaesthetic trainees experienced in the technique. The fascia iliaca compartment was localised superficial to the iliopsoas muscle using Sonosite M-Turbo ultrasound machine (Sonosite Inc., Bothell, USA) with an L38x 10-5 $\mathrm{MHz} 38 \mathrm{~mm}$ broadband linear array probe. $30 \mathrm{ml}$ of $0.25 \%$ levobupivacaine was introduced into the fascial plane just below fascia iliaca via $18 \mathrm{G}$ Tuohy $100 \mathrm{~mm}$ needle using an in-plane approach. The dose of $30 \mathrm{ml}$ of $0.25 \%$ levobupivacaine was standardised for the institution in which the study was undertaken and is similar in terms of volume, drug and dosing with multiple 
published dosing regimens for FICB [7-11]. All local anaesthetics injections were confirmed on ultrasound as being delivered into the correct fascial plane.

Blood samples were obtained by aspiration from a venous cannula in the basilic or cephalic vein. Venous blood samples were taken at 10, 20, 30, 45, 60, 75, 90, 105, 120 and 240 minutes following injection of local anaesthetic.

Patients were continuously monitored by nursing staff for the presence of early symptoms of central nervous system excitation associated with local anaesthetic toxicity (perioral tingling, metallic taste, light-headedness, blurred vision, tinnitus or drowsiness) and for later signs of cardiovascular depression, seizures or muscle twitching. ECG and non-invasive blood pressure monitoring was maintained throughout. Pain scores were measured using a standard numeric rating scale (NRS-11; where 0 is no pain, and 10 is the worst pain imaginable) at rest and on 15 degree straight leg raise at the same time intervals as blood sampling.

The protocol for this study was approved by the London - Central Research Ethics Committee (REC reference 16/LO/1321) and Health Research Authority.

\subsection{End points and measurements}

Blood samples were centrifuged immediately after sampling at the study site before freezing and storage at $-80^{\circ} \mathrm{C}$. Plasma samples were then transferred to the Analytic Services International Laboratory at St. George's University of London. Levobupivacaine was extracted from 100 $\mu \mathrm{L}$ of human plasma by solvent extraction, before addition to an internal standard $\left(0.5 \mu \mathrm{g} . \mathrm{ml}^{-1}\right.$ ropivacaine solution), $100 \mu \mathrm{L}$ of $1 \mathrm{M}$ sodium hydroxide and $3 \mathrm{ml}$ methyl tert-butyl ether (MTBE). The samples underwent sequential centrifugation before being submitted for analysis on Shimadzu QP2010 standard gas chromatograph-mass spectrometer system.

Concentrations of levobupivacaine in calibration standards and quality control samples were calculated using peak area ratio of analyte and internal standard. The calibration lines were generated using a linear regression line with a weighting of $1 / \mathrm{x}^{2}$. The imprecision of the assay, as assessed by the coefficient of variation, was $\leq 5 \%$ at 0.19 and $0.76 \mu \mathrm{g} \cdot \mathrm{ml}^{-1}$.

The concentrations of human $\alpha 1$ acid glycoprotein in plasma was determined by enzyme-linked immunosorbent assay (ELISA) with colorimetric detection using a micro titre plate reader 
(A1BG ELISA Kit ABIN512507, BMG LabTech). The coefficient of variation of the assay was $<14 \%$ at $0.3 \mathrm{mg} \cdot \mathrm{ml}^{-1}$, and $<9 \%$ at $1.5 \mathrm{mg} \cdot \mathrm{ml}^{-1}$.

\subsection{Statistical analysis}

Data were analysed with descriptive statistics (medians, IQR [range], means, standard deviations, and \% accuracy), including assessment of the calibration standards and quality control samples. Correlation was analysed using Pearson's test. The following parameters were reported: maximum plasma drug concentration $\left(\mathrm{C}_{\max }, \mu \mathrm{g} \cdot \mathrm{ml}^{-1}\right)$ and the time to reach $\mathrm{C}_{\max }\left(\mathrm{t}_{\max }\right.$, mins). Area under the plasma concentration curve until the last measurable concentration $\left(C_{n}\right)$, was calculated by the linear trapezoidal method $\left(\mathrm{AUC}_{\mathrm{t}}, \mu \mathrm{g} \times \mathrm{h} \cdot \mathrm{ml}^{-1}\right)$ [12]. 


\section{Results}

Data collection took place from November 2016 to November 2017, during which 12 patients gave written and informed consent for inclusion in the study. Patient recruitment is shown in Figure 1. All patients had fractured neck of femur confirmed on radiograph, with baseline characteristics summarised in Table 1. Venous blood samples were collected from all 12 patients at each of the 10 time indices, although one blood sample (10 minutes, from participant F) was damaged and unavailable for analysis purposes. A total of 119/120 (99.2\%) of all plasma samples were therefore analysed for total levobupivacaine concentration. All patients received the same standard dose of $30 \mathrm{ml} 0.25 \%$ levobupivacaine; equivalent to a total dose of $75 \mathrm{mg}$ levobupivacaine.

Change in plasma levobupivacaine concentrations over time is shown in Figures 2 and 3. Total plasma levobupivacaine $\mathrm{C}_{\max }$ concentration had a median (IQR) of $0.82 \mu \mathrm{g} \cdot \mathrm{ml}-1$ (0.47 - 1.03) and mean (SD) of $0.84 \mu \mathrm{g} \cdot \mathrm{ml}^{-1}(0.39)$, and $\mathrm{t}_{\max }$ occurred at a median 45 minutes [IQR, 41:20 60:00]. The highest individual peak plasma concentration was $1.42 \mu \mathrm{g} . \mathrm{ml}^{-1}$, occurring at $60 \mathrm{~min}$ after FICB injection. Mean area under curve $\left(\mathrm{AUC}_{\mathrm{t}}\right)$ for levobupivacaine plasma concentration was $153.28 \mu \mathrm{g} \times \mathrm{h} \cdot \mathrm{ml}^{-1}(75.38)$.

Patients had a median age of 89 years (IQR, $83-91$ ). Total dose of levobupivacaine per patient was equivalent to a median of $1.39 \mathrm{mg} . \mathrm{kg}^{-1}$ (IQR, $\left.1.14-1.50\right)$.

No patients exhibited symptoms or signs consistent with local anaesthetic toxicity, mild or otherwise over the study monitoring period. No adverse ECG or haemodynamic effects were observed in any patient. No complications or adverse events following FICB were reported, even after the study monitoring period had finished. $\alpha 1$ acid glycoprotein plasma concentration had a median $0.88 \mathrm{mg} \cdot \mathrm{ml}^{-1}$ (IQR, $0.79-1.21$ ). There was no significant correlation between plasma $\alpha 1$ acid glycoprotein concentration and levobupivacaine $\mathrm{C}_{\max }(\mathrm{r}=0.11 ; \mathrm{p}=0.75)$. Nor was any significant association found between $\mathrm{C}_{\max }$ and weight or BMI.

Analgesic effectiveness of FICB was measured using pain scores at the same as blood sampling. Pain scores at rest and on 15 degree straight leg raise are shown in figure 4. All patients experienced a reduction in baseline pain following FICB, although one patient (A) had an increase in pain scores during passive leg raise from 5 to 8 between 120 and 240 minutes. 
Median pain scores at rest reduced from $3(\mathrm{IQR}, 0-4)$ to $0(\mathrm{IQR}, 0-0)$ by 45 minutes (i.e. $\left.\mathrm{t}_{\max }\right)$. Over the same time period pain scores during 15 degree straight leg raise reduced from 5 (IQR, 3 $-5.5)$ to $2(\mathrm{IQR}, 0.5-4)$. 


\section{Discussion}

This pharmacokinetic analysis of plasma local anaesthetic concentration shows that FICB in elderly patients exhibit a slow absorption process that leads to delayed $t_{\max }$ and low $\mathrm{C}_{\max }$. To date, no prior study has described the pharmacokinetic properties of local anaesthetic administered via FICB in elderly patients.

Patients with fractured neck of femur (the most common recipients of FICBs) are often elderly and frail, with a median age of 84 years for men and 83 years for women in the UK [13]. Reports of toxicity with levobupivacaine are rare and most cases of systemic toxicity of local anaesthetics are as a result of accidental intravascular injection. However age-related pathophysiological changes may increase the susceptibility to amide local anaesthetic toxicity and many patients with hip fracture also have impaired cognitive function, which can present challenges for reliably detecting early clinical evidence of central nervous system adverse effects.

Levobupivacaine is long-acting amide local anaesthetic with a clinical profile closely matching that of bupivacaine. However, current preclinical safety and toxicity data show an advantage for levobupivacaine over bupivacaine [14-16]. Levobupivacaine is the pure S(-) enantiomer of racemic bupivacaine. Although the drug is highly protein bound ( $>97 \%$, primarily to $\alpha 1$ acid glycoprotein) we did not find any association between $\alpha 1$ acid glycoprotein and total levobupivacaine concentrations. $11 / 12$ of the $\alpha 1$ acid glycoprotein concentrations were found to be within standard reference ranges for young, healthy adults $\left(0.4-1.4 \mathrm{mg}^{-\mathrm{ml}^{-1}}\right)$, despite being measured in the context of the acute, painful stress of a fractured neck of femur. It is possible that as patients were all pre-operative and bloods were taken within 12 hours of presentation to hospital, the acute phase rise in plasma protein concentration may not have been captured at time of blood sampling.

Two previous studies have examined local anaesthetic pharmacokinetics following single shot FICB, but both including only children. A study using $2 \mathrm{mg} \cdot \mathrm{kg}^{-1}$ of $0.25 \%$ bupivacaine in nine patients aged 1-14 years found a median $\mathrm{C}_{\max }$ of $1.1 \mu \mathrm{g} \cdot \mathrm{ml}^{-1}$ (range 0.54-1.29 $\mu \mathrm{g} \cdot \mathrm{ml}^{-1}$ ) and median $t_{\max }$ of $45 \min [16]$. Lower median values were found in a similar group randomly assigned to $2 \mathrm{mg} . \mathrm{kg}^{-1}$ bupivacaine with 1:200,000 epinephrine $\left(\mathrm{C}_{\max }\right.$ of $0.35 \mu \mathrm{g} \cdot \mathrm{ml}^{-1}$ and $\mathrm{t}_{\max } 20$ mins). A second small study of children, aged $1-15$ years, receiving $3.5 \mathrm{mg} . \mathrm{kg}^{-1}$ ropivacaine for 
FICB found high $\mathrm{C}_{\max }$ of $4.33-5.60 \mu \mathrm{g} \cdot \mathrm{ml}^{-1}$ in those receiving $0.5 \%$ ropivacaine [17]. No signs of toxicity were observed in the study.

A single study of ten >80 year old hip fracture patients following a femoral 3 -in-1 nerve block, injected at similar anatomical sites to a FICB, showed a mean $\mathrm{C}_{\max }$ of $0.74 \mu \mathrm{g} \cdot \mathrm{ml}^{-1}(0.64)$ after $2 \mathrm{mg} \cdot \mathrm{kg}^{-1}$ bupivacaine [18]. Significant variation was identified, with a highest plasma level of $1.83 \mu \mathrm{g} \cdot \mathrm{ml}^{-1}$ detected. All patients had arterial blood sampled during surgery under general anaesthesia, eliminating the ability to reliably identify signs and symptoms of local anaesthetic toxicity. Concurrent blood loss and intravenous fluid administration are unquantified variables that may confound interpretation of these results, by altering plasma concentrations of solutes.

Early presentation of levobupivacaine toxicity manifests with central nervous systems symptoms of disorientation, drowsiness, tinnitus, numbness of the tongue, slurred speech, and, ultimately, tonic-clonic seizures [19-24]. Clinical studies in young, healthy adult volunteers have evaluated tolerance of progressively increasingly doses of levobupivacaine via intravenous infusion, terminated at the occurrence of early central nervous system symptoms or changes in cardiac function. In such studies toxic effect begin to manifest at levobupivacaine plasma concentrations of approximately 2.4 - 2.6 $\mu \mathrm{g} \cdot \mathrm{ml}^{-1}$ [24-26]. Case reports of severe systematic toxicity and measured plasma concentration of levobupivacaine are scarce, but support human experimental data; with a serum concentration of levobupivacaine of $2.7 \mathrm{mg} \cdot \mathrm{ml}^{-1}$ measured 14 minutes after inadvertent intravenous injection associated with central nervous system symptoms [19] in one patient.

Our study was conducted in ideal clinical conditions, in pre-operative patients who were not undergoing any significant fluid resuscitation. Results show a similar pharmacokinetic pattern to levobupivacaine injection into the fascia iliaca compartment as with femoral 3-in-1 blocks in elderly patients, albeit $t_{\max }$ occurred more quickly (99 minutes following 3-in-1 block; 45 minutes after FICB) and with less variability in individual $\mathrm{C}_{\max }$ identified. Alongside total plasma concentrations, a rapid rate of increase in local anaesthetic plasma concentration may also be related to toxicity. Previous studies of nerve block injections around more vascular anatomical structures using ultrasound and non-ultrasound guided techniques have demonstrated much more rapid rises to $t_{\max }, 7.5 \mathrm{~min}$ following paravertebral block [27], 10min following interscalene block [28] and 30min following transverse abdominus plane (TAP) block [29]. All 
these pharmacokinetic investigations were conducted in young adults, rather than elderly patients.

Only total, rather than bound and unbound concentrations of levobupivacaine were measured in this study. The free or unbound fraction of an amide local anaesthetic may be more predictive of toxicity than the total concentration. All patients had meticulous clinical monitoring to detect even subtle signs of toxicity, with none identified. The study had a sample size of twelve patients, but all were representative in terms of age and frailty with the cohort that FICB are most commonly provided to in clinical practice. Conclusions must be carefully drawn, accepting that pharmacokinetics are typically log-normally distributed and that some patients may (rarely) experience much higher concentrations than were recorded in the study. Patients with advanced cognitive decline or delirium were not recruited to the study. It is possible that such patients may have a reduced threshold to local anaesthetic central nervous system toxicity than patients with normal cognitive function.

\section{Conclusion}

This study provides clinical and pharmacokinetic evidence that administration of a $75 \mathrm{mg}$ dose of levobupivacaine in a FICB to elderly patients using ultrasound guidance results in peak plasma concentrations below the reputed toxic threshold and provides effective, safe pain management for fractured neck of femur. The findings contribute to a growing body of evidence that local anaesthetic pharmacokinetics are both technique and patient demographic specific - being related to anatomical location of the injectate and age-related patient characteristics. Further research is required to quantify the exact thresholds for local anaesthetic toxicity in elderly patients, the safe range of doses for FICB and techniques that can be employed to limit systemic local anaesthetic absorption after FICB. 


\section{Acknowledgements}

We wish to thank Toqa El-Nahhas for her assistance in processing the blood sample analysis at the Analytic Services International Laboratory.

\section{Compliance with Ethical Standards}

Funding Supported by a National Institute of Academic Anaesthesia (NIAA) and Regional Anaesthesia-UK (RA-UK) research grant.

Conflict of interest Atholl Johnson is the Facility Director at Analytical Services International Ltd, St George's University of London, where all sample analysis was conducted. The authors declare no other conflicts of interest.

Ethical approval The study was approved by the London - Central Research Ethics Committee (REC reference 16/LO/1321) and Health Research Authority. The study was conducted according to the Helsinki Declaration, ICH Good Clinical Practice recommendations and applicable local regulations.

Informed consent Written informed consent was received from all subjects prior to the conduct of any study-related procedures. 


\section{References}

1. Dalens B, Vanneuville G, Tanguy A. Comparison of the fascia iliaca compartment block with the 3-in-1 block in children. Anesth Analg. 1989;69:705-713.

2. Foss N, Kristensen BB, Bundgaard M, et al H. Fascia iliaca compartment blockade for acute pain control in hip fracture patients. Anesthesiology 2007;106:773-778.

3. Abernethy $\mathrm{D}$, Greenblatt $\mathrm{D}$. Impairment of lidocaine clearance in elderly male subjects. $J$ Cardiovasc Pharmacol. 1983;5:1093-1096.

4. Muravchick S. Geroanesthesia: principles for the management of the elderly patient. Mosby, St. Louis, USA. 1997:306.

5. Muravchick S. The aging process: anesthetic implications. Acta Anaesthesiol Belg. 1998;49:85-90.

6. Simon MJ, Veering BT, Stienstra R, van Kleef JW, Burm AG. Effect of age on the clinical profile and systemic absorption and disposition of levobupivacaine after epidural administration. Br J Anaesth. 2004;93:512-520.

7. Newman B, McCarthy L, Thomas PW, May P, Layzell M, Horn K. A comparison of preoperative nerve stimulator-guided femoral nerve block and fascia iliaca compartment block in patients with a femoral neck fracture. Anaesthesia. 2013;68:899-903.

8. Haines L, Dickman E, Ayvazyan S et al. Ultrasound-guided fascia iliaca compartment block for hip fractures in the emergency department. J Emerg Med. 2012;43:692-697.

9. Godoy Monzón D, Iserson KV, Vazquez JA. Single fascia iliaca compartment block for posthip fracture pain relief. J Emerg Med. 2007;32:257-262.

10. New York School of Regional Anaesthesia. Ultrasound-Guided Fascia Iliaca Block. 2013. Available at: https://www.nysora.com/ultrasound-guided-fascia-iliaca-block-2. Accessed 26th September 2018.

11. Stevens M, Harrison G, McGrail M. A modified fascia iliaca compartment block has significant morphine-sparing effect after total hip arthroplasty. Anaesth Intensive Care. 2007;35:949-952. 
12. Proost JH, Meijer DKW. MW/Pharm, an integrated software package for drug dosage regimen calculation and therapeutic drug monitoring. Comp Biol Med. 1992;22:155-162.

13. National Institute for Health and Care Excellence. Clinical Guideline 124: the management of hip fracture in adults, August 2011. Available at: https://www.nice.org.uk/guidance/cg124. Accessed 26th September 2018.

14. Foster RH, Markham A. Levobupivacaine: a review of its pharmacology and use as a local anaesthetic. Drugs. 2000;59:551-579.

15. Sanford M, Keating G. Levobupivacaine: a review of its use in regional Anaesthesia and pain management. Drugs. 2010;70:761-791.

16. Paut O, Schreiber E, Meyrieux V et al. High plasma ropivacaine concentrations after fascia iliaca compartment block in children. Br J Anaesth. 2004;92:416-418.

17. Doyle E, Morton N, McNicol L. Plasma bupivaciane levels after fascia iliaca block with and without adrenaline. Paediatr Anaesth 1997;7:121-124.

18. Snoeck MM, Vree TB, Gielen MJ. Steady state bupivacaine plasma concentrations and safety of a femoral " 3 -in-1" nerve block with bupivacaine in patients over 80 years of age. Int J Clin Pharmacol Ther. 2003;41:107-113.

19. Kopacz DJ, Allen HW. Accidental intravenous levobupivacaine. Anesth Analg. 1999;89:1027-1029.

20. Pirotta D, Spriqqe J. Convulsions following axillary brachial plexus blockade with levobupivacaine. Anaesthesia 2002;57:1187-1189.

21. Khan H, Atanassoff PG. Accidental intravascular injection of levobupivacaine and lidocaine during the transarterial approach to the axillary brachial plexus. Can J Anaesth 2003; 50: 95.

22. Crews JC, Rothman TE. Seizure after levobupivacaine for interscalene brachial plexus block. Anesth Analg. 2003;96:1188-1190.

23. Breslin DS, Martin G, Macleod DB, D'ercole F, Grant SA. Central nervous system toxicity following the administration of levobupivacaine for lumbar plexus block: A report of two cases. Reg Anesth Pain Med. 2003;28:144-147. 
24. Knudsen K, Suurküla B, Blomberg S, Sjövall J, Edvardsson N. Central nervous and cardiovascular effects of i.v. infusions of ropivacaine, bupivacaine and placebo in volunteers. Br J Anaesth. 1997;78:507-514.

25. Gristwood R, Bardsley H, Baker H, Dickens J. Reduced cardiotoxicity of levobupivacaine compared with racemic bupivacaine (Marcaine): new clinical evidence. Expert Opin Investig Drugs. 1994;3:1209-1212.

26. Bardsley H, Gristwood R, Baker H, Watson N, Nimmo W. A comparison of the cardiovascular effects of levobupivacaine and rac-bupivacaine following intravenous administration to healthy volunteers. Br J Clin Pharmacol. 1998;46:245-249.

27. Karmakar MK, Ho AM, Law BK, Wong AS, Shafer SL, Gin T. Arterial and venous pharmacokinetics of ropivacaine with and without epinephrine after thoracic paravertebral block. Anesthesiology 2005;103:704-711.

28. Rettig HC, Lerou JG, Gielen MJ, Boersma E, Burm AG. The pharmacokinetics of ropivacaine after four different techniques of brachial plexus blockade. Anaesthesia 2007;62:1008-1014.

29. Griffiths JD, Barron FA, Grant S, Bjorksten AR, Hebbard P, Royse CF. Plasma ropivacaine concentrations after ultrasound-guided transversus abdominis plane block. Br J Anaesth. 2010;105:853-856. 
Table 1: Summary of baseline characteristics of the included patients. Mean (SD) unless specified.

\begin{tabular}{|c|c|}
\hline Variable & Results $(n=12)$ \\
\hline Median age (IQR [range]) & $88.5(83-91[82-100])$ \\
\hline Gender (male/female) & 2 male / 10 female \\
\hline Height (m) & $1.60 \mathrm{~m}(0.10)$ \\
\hline Weight (kg) & $59.6(13.6)$ \\
\hline $\mathrm{BMI}\left(\mathrm{kg} \cdot \mathrm{m}^{-2}\right)$ & $23.3(5.1)$ \\
\hline $\begin{array}{l}\text { Estimated glomerular filtration rate } \\
\left(\mathrm{mL} / \mathrm{min} / 1.73 \mathrm{~m}^{2}\right)^{\mathrm{a}}\end{array}$ & $46.7(23.2)$ \\
\hline Plasma albumin $\left(\mathrm{g} \cdot \mathrm{L}^{-1}\right)$ & $37.8(3.3)$ \\
\hline Plasma alpha-1 glycoprotein (mg.ml $\left.{ }^{-1}\right)$ & $0.96(0.36)$ \\
\hline
\end{tabular}




\section{Figure Legends}

\section{Fig. 1}

Flowchart of patient recruitment and participation

Fig. 2:

Mean plasma total levobupivacaine concentrations following fascia iliaca block. Error bars are SD

\section{Fig. 3}

Individual patient total levobupivacaine concentrations following fascia iliaca block

\section{Fig. 4}

Box plot of pain scores at rest and on 15 degree straight leg raise at time indexes from 10 to 120 minutes. Measuring using numeric rating scale (NRS-11). Solid line = median; box = IQR; whisker = upper and lower range 


\section{Allocation}

\section{Follow-Up}

Lost to follow-up $(n=0)$

Discontinued intervention $(n=0)$

Excluded $(n=18)$

- Not meeting inclusion criteria $(n=12)$

- Declined to participate $(n=6)$

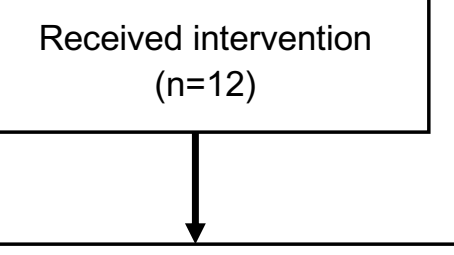




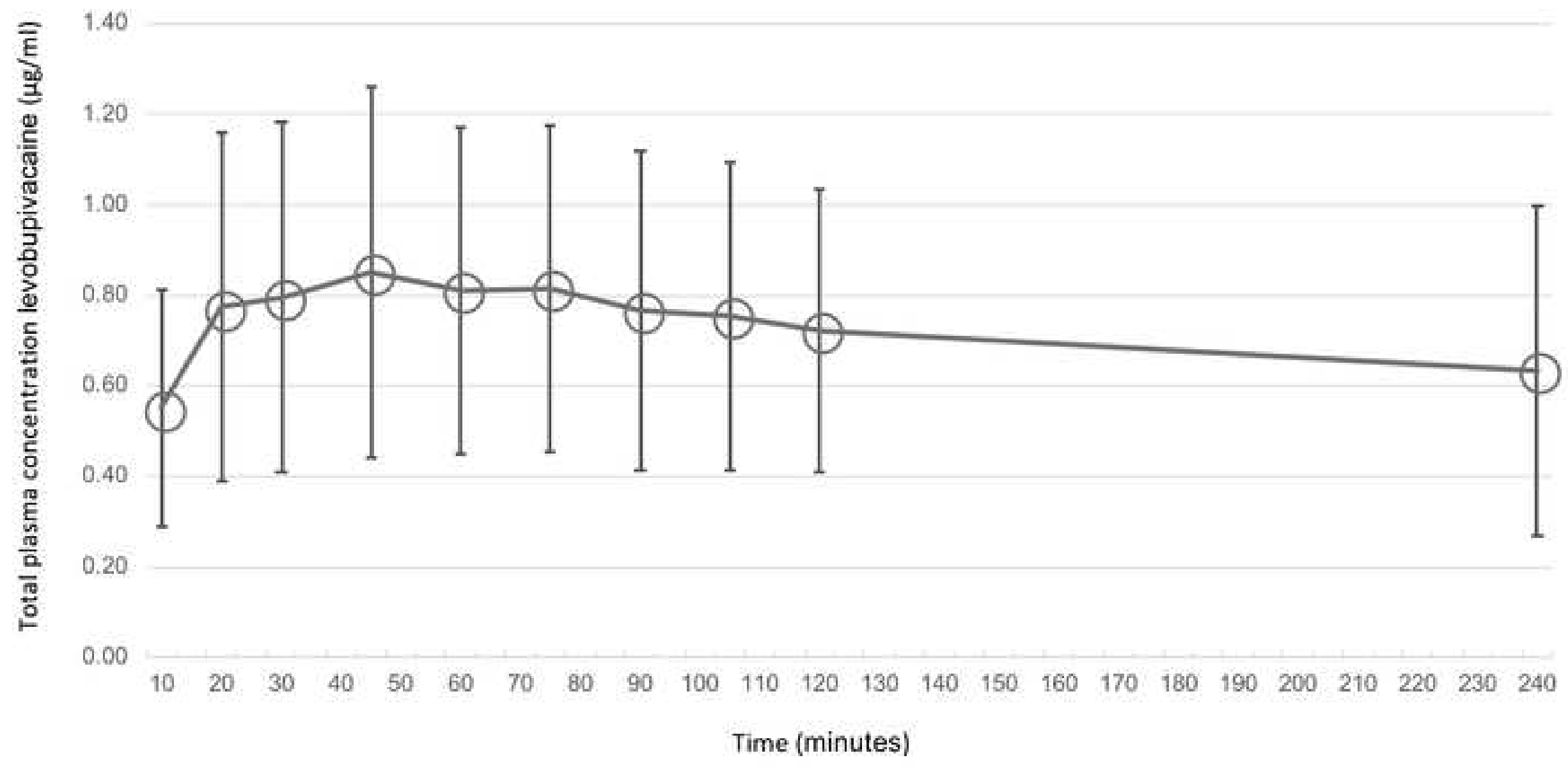




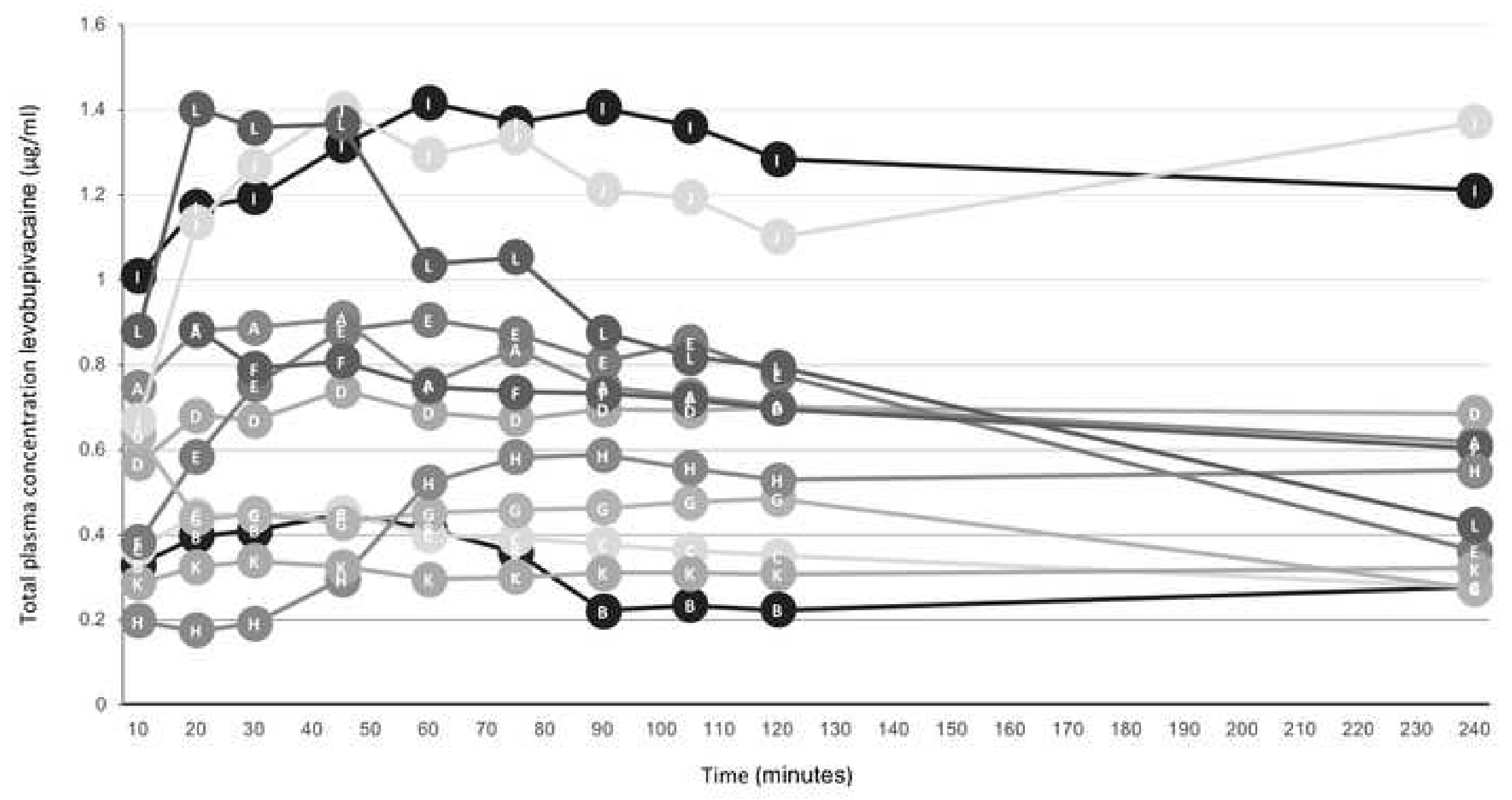




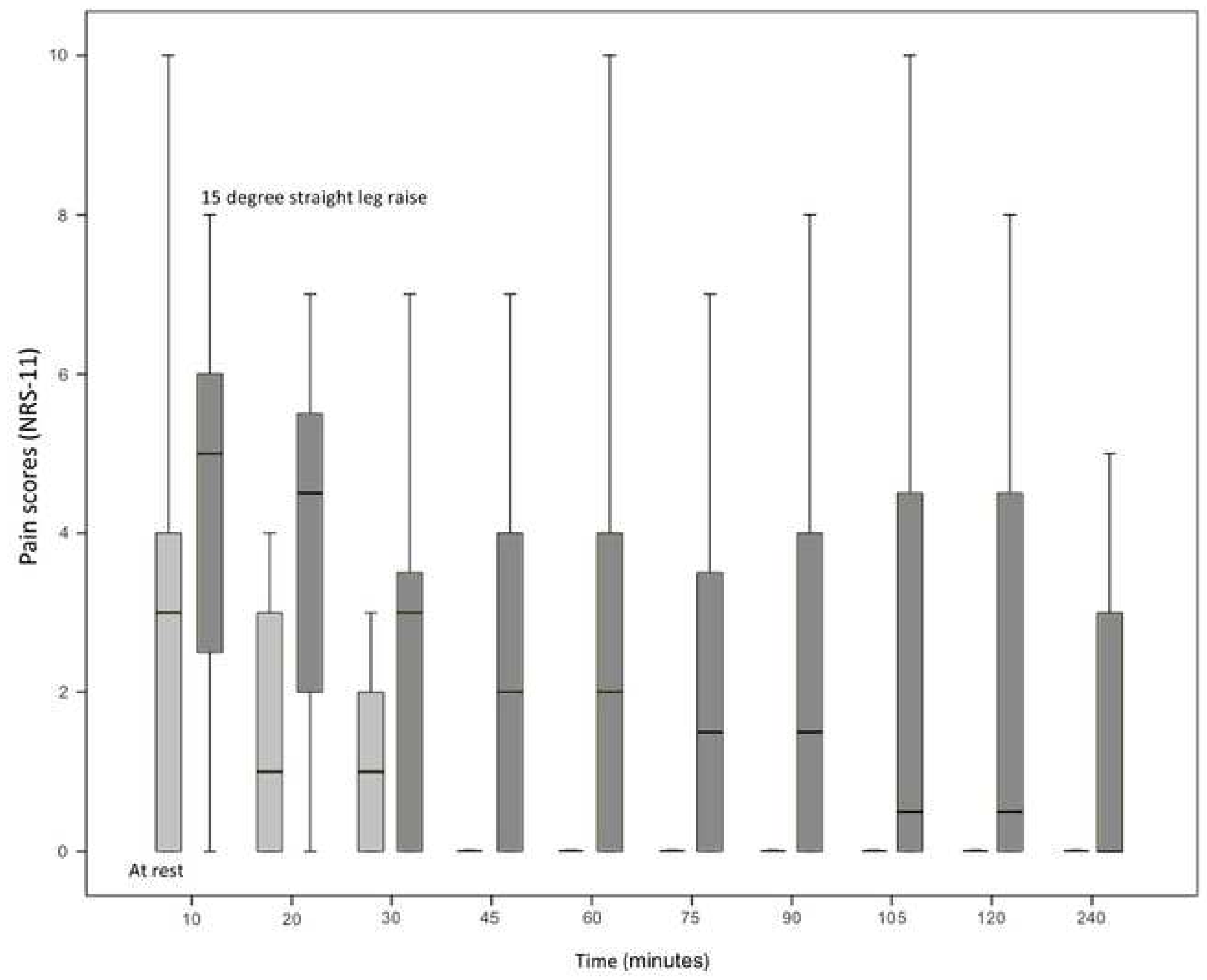

